TITLE:

\title{
Thermodynamic Entropy as a Noether Invariant.
}

\author{
$\operatorname{AUTHOR}(S)$ :
}

Sasa, Shin-Ichi; Yokokura, Yuki

\section{CITATION:}

Sasa, Shin-Ichi ... [et al]. Thermodynamic Entropy as a Noether Invariant.. Physical review letters 2016, 116(14): 140601.

ISSUE DATE:

2016-04-08

URL:

http://hdl.handle.net/2433/212493

RIGHT:

(c) 2016 American Physical Society 


\title{
Thermodynamic Entropy as a Noether Invariant
}

\author{
Shin-ichi Sasa \\ Department of Physics, Kyoto University, Kyoto 606-8502, Japan \\ Yuki Yokokura \\ International Centre for Theoretical Sciences, Survey No. 151, Shivakote, Hesaraghatta Hobli, Bengaluru North 560 089, India \\ iTHES Research Group, RIKEN, Wako, Saitama 351-0198, Japan
}

(Received 8 October 2015; published 7 April 2016)

\begin{abstract}
We study a classical many-particle system with an external control represented by a time-dependent extensive parameter in a Lagrangian. We show that thermodynamic entropy of the system is uniquely characterized as the Noether invariant associated with a symmetry for an infinitesimal nonuniform time translation $t \rightarrow t+\eta \hbar \beta$, where $\eta$ is a small parameter, $\hbar$ is the Planck constant, $\beta$ is the inverse temperature that depends on the energy and control parameter, and trajectories in the phase space are restricted to those consistent with quasistatic processes in thermodynamics.
\end{abstract}

DOI: 10.1103/PhysRevLett.116.140601

Introduction.-Entropy is a fundamental concept in physics. It appears in thermodynamics [1,2], statistical mechanics [3], information theory [4], computation theory [5], quantum information theory [6], and thermodynamics of black holes [7]. Recently, the interrelation between different types of entropy has been discovered. The second law of thermodynamics has been extended so as to apply systems with a feedback control through the exchange of information, not of energy, between the system and the controller [8]. This opens up studies in the intersection of thermodynamics and information theory [9]. As another development, there have been attempts to connect black hole entropy to entanglement entropy $[10,11]$, and in the anti-de Sitter/conformal field theory context a novel notion of holographic entanglement entropy has appeared, which provides a dual description between boundary entanglement entropy and dynamics of bulk spacetime [12]. By synthesizing various aspects of entropy, we thus obtain a deeper understanding of fundamental laws in physics. Now, there is a paper [13] which claims that black hole entropy is obtained as the Noether charge associated with the horizon Killing field. We are then naturally led to ask whether thermodynamic entropy of standard materials is also characterized by a Noether invariant.

Suppose that we have a many-particle isolated system in a box, and that an external controller moves a piston, which may be described by a time-dependent single-body potential. Then, in response to the fact that thermodynamic entropy keeps a constant value in quasistatic adiabatic processes [1], it was proved that along almost all the solution trajectories to the equation of motion with quasistatic change in the volume, the phase-space volume enclosed by the energy surface including the phase-space point at time $t$ is invariant [14-19]. Thus, the logarithm of the phase-space volume provides a definition of timedependent entropy in mechanics. The main result of this
Letter is that there exists a symmetry by which the entropy is uniquely characterized as a Noether invariant.

The key step in our theory is to formulate a special class of trajectories that are consistent with quasistatic processes in thermodynamics. By restricting the domain of the action to this class of trajectories, we find a symmetry for an infinitesimal nonuniform time translation $t \rightarrow t+\eta \hbar \beta$, where $\eta$ is a small parameter, $\hbar$ is the Planck constant, and $\beta$ is the inverse temperature determined by applying the thermodynamic relation to the time-dependent entropy. It should be noted that our theory stands on classical mechanics, classical statistical mechanics, and thermodynamics, and thus the Planck constant does not appear. Nevertheless, our theory leads to the existence of a universal constant with the same dimension as the action.

Below, we first describe a setting up of classical mechanics of the particle system, and discuss a generalized Noether theorem associated with a symmetry. We then define trajectories consistent with quasistatic processes based on statistical mechanics. By combining these two concepts, we derive our main result.

Mechanics.-Let $q(t) \in \mathbb{R}^{3 N}$ be a collection of coordinates of $N$ particles with short-range interaction in a box of volume $V$. We particularly focus on macroscopic systems where the extensive behavior is observed for large $N$. We denote the trajectory $(q(t))_{t=t_{i}}^{t_{f}}$ by $\hat{q}$. We also introduce an extensive control parameter $\alpha$, whose typical example is the volume $V$. (Formally, $\alpha$ is a complete set of extensive work variables.) For a fixed protocol of the parameter $\hat{\alpha}=(\alpha(t))_{t=t_{i}}^{t_{f}}$, the action $\mathcal{I}(\hat{q}, \hat{\alpha})$ is given by

$$
\mathcal{I}(\hat{q}, \hat{\alpha})=\int_{t_{i}}^{t_{f}} d t L(q(t), \dot{q}(t), \alpha(t)),
$$

where the dot denotes the time derivative. All the mechanical properties are represented by the Lagrangian [20]. We also assume that there is no conserved quantity other than 
the total energy for the system with $\alpha$ fixed, $E(q, \dot{q}, \alpha)=$ $\dot{q} \partial L / \partial \dot{q}-L(q, \dot{q}, \alpha)$.

We consider a nonuniform time translation: $t \rightarrow t^{\prime}=$ $t+\eta \xi(q, \dot{q}, \alpha)$. Here, $\eta$ is a small parameter, and the functional form of $\xi$ is not specified yet. Then, the transformation $\hat{q} \rightarrow \hat{q}^{\prime}$ is given by $q^{\prime}\left(t^{\prime}\right)=q(t)$, because the position of particles is independent of relabeling time coordinate. The transformation $\hat{\alpha} \rightarrow \hat{\alpha}^{\prime}$ corresponds to $\alpha^{\prime}\left(t^{\prime}\right)=\alpha\left(t^{\prime}\right)$, because the protocol $\hat{\alpha}$ is fixed. We represent this transformation by index $G$, and neglect the contribution of $O\left(\eta^{2}\right)$. Then, the change in action $\delta_{G} \mathcal{I} \equiv \mathcal{I}\left(\hat{q}^{\prime}, \hat{\alpha}^{\prime}\right)-$ $\mathcal{I}(\hat{q}, \hat{\alpha})$ is expressed as

$$
\delta_{G} \mathcal{I}=\int_{t_{i}}^{t_{f}} d t\left[\bar{\delta}_{G} L+\eta \frac{d(\xi L)}{d t}\right],
$$

where we have defined $\bar{\delta}_{G} L \equiv L\left(q^{\prime}(t), \dot{q}^{\prime}(t), \alpha^{\prime}(t)\right)$ $L(q(t), \dot{q}(t), \alpha(t))$. Noting that $\bar{\delta}_{G} q(t) \equiv q^{\prime}(t)-q(t)=$ $-\eta \xi \dot{q}$ and introducing the Euler-Lagrange derivative

$$
\mathcal{E} \equiv \frac{\partial L}{\partial q}-\frac{d}{d t} \frac{\partial L}{\partial \dot{q}},
$$

we express $\bar{\delta}_{G} L$ in terms of $\bar{\delta}_{G} q$. Thus, we obtain

$$
\delta_{G} \mathcal{I}=\eta \int_{t_{i}}^{t_{f}} d t\left\{-\mathcal{E} \dot{q} \xi+\frac{d}{d t}\left[\xi\left(L-\dot{q} \frac{\partial L}{\partial \dot{q}}\right)\right]\right\} .
$$

Now suppose that, for some $\hat{\alpha}$, there exist $\xi(q, \dot{q}, \alpha)$ and $\psi(q, \dot{q}, \alpha)$ such that $[21,22]$

$$
\delta_{G} \mathcal{I}=\eta \int_{t_{i}}^{t_{f}} d t \frac{d \psi}{d t}
$$

for a class of trajectories $\hat{q}$, which is identified later. Then, (4) is written as

$$
\int_{t_{i}}^{t_{f}} d t \mathcal{E} \dot{q} \xi=-\left.(\psi+E \xi)\right|_{t_{i}} ^{t_{f}}
$$

This leads to two important properties. First, because $\mathcal{E}=0$ at any solution $\hat{q}_{*}$, we obtain a conservation law

$$
\left.\left(\psi_{*}+E_{*} \xi_{*}\right)\right|_{t_{i}} ^{t_{f}}=0 .
$$

Here, the subscript of $B_{*}$ represents the evaluation of a quantity $B$ at a solution trajectory $q_{*}(t)$. Second, by substituting $q(t)=q_{*}\left(t+\eta \xi_{*}\right)$ into (6), we have

$$
\left.\int_{t_{i}}^{t_{f}} d t \mathcal{E} \dot{q} \xi\right|_{q=q_{*}\left(t+\eta \xi_{*}\right)}=-\left.\left.(E \xi+\psi)\right|_{t_{i}^{\prime}} ^{t_{f}^{\prime}}\right|_{*}
$$

where we have used $q_{*}\left(t_{i}+\eta \xi_{*}\left(t_{i}\right)\right)=q_{*}\left(t_{i}{ }^{\prime}\right)$. Because the conservation law (7) holds for any $t_{i}$ and $t_{f}$, the right-hand side of (8) is equal to zero. Expanding the left-hand side with respect to $\eta$, we obtain

$$
\left.\int_{t_{i}}^{t_{f}} d t \frac{\delta \mathcal{E}}{\delta q}\left(\bar{\delta}_{G} q\right) \dot{q} \xi\right|_{*}=0
$$

where we have used the equation of motion $\left.\mathcal{E}\right|_{*}=0$. The relation (9) implies that $q_{*}+\left.\bar{\delta}_{G} q\right|_{*}$ is a solution of the same equation of motion [21]. That is, the transformation $G$ maps each solution trajectory to another one in the system $\mathcal{I}(\hat{q}, \hat{\alpha})$. This property was referred to as a dynamical symmetry $[25,26]$. If $\psi$ in (5) is independent of $\dot{q}$, which includes the case $\psi=0, \mathcal{I}\left(\hat{q}^{\prime}, \hat{\alpha}^{\prime}\right)$ provides the same equation of motion as that for $\mathcal{I}(\hat{q}, \hat{\alpha})$. In a more general case where $\psi$ depends on $\dot{q}$, the action $\mathcal{I}\left(\hat{q}^{\prime}, \hat{\alpha}^{\prime}\right)$ defines a different dynamical system. Even for this case, however, (5) represents a symmetry, leading to the dynamical symmetry and the conservation law (7), as we have seen above. This was called a generalized Noether theorem [27]. In this context, $\psi+E \xi$ is the Noether invariant associated with the transformation $G$.

Thermodynamics.-Let us briefly review statistical mechanics. We introduce a phase-space coordinate $\Gamma=$ $(q, p)$ with the momentum $p \equiv \partial L / \partial \dot{q} \in \mathbb{R}^{3 N}$, and assume that $\dot{q}$ can be uniquely determined for $(q, p)$. Then, $H(\Gamma, \alpha)=E(q, \dot{q}(q, p), \alpha)$ is the Hamiltonian. The expectation of any quantity $A(\Gamma)$ with respect to the microcanonical ensemble of $(E, \alpha)$ is defined as

$$
\langle A\rangle_{E, \alpha}^{\mathrm{mc}} \equiv \frac{1}{\Sigma(E, \alpha)} \int d \Gamma \delta(E-H(\Gamma, \alpha)) A(\Gamma),
$$

where $\Sigma(E, \alpha) \equiv \int d \Gamma \delta(E-H(\Gamma, \alpha))$ is the normalization constant. Throughout this Letter, the Boltzmann constant is set to unity. According to the formula in statistical mechanics, the entropy $S$ is defined as

$$
S(E, \alpha) \equiv \log \frac{\Omega(E, \alpha)}{N !},
$$

with $\Omega(E, \alpha) \equiv \int d \Gamma \theta(E-H(\Gamma, \alpha))$, where $\theta(x)=1$ for $x \geq 0$ and $\theta(x)=0$ for $x<0$ [29]. We can then confirm the fundamental relation in thermodynamics [30]:

$$
d S=\beta d E-\beta\left\langle\frac{\partial H}{\partial \alpha}\right\rangle_{E, \alpha}^{\mathrm{mc}} d \alpha
$$

with the definition of the inverse temperature

$$
\beta \equiv \frac{\Sigma(E, \alpha)}{\Omega(E, \alpha)} .
$$

When $\alpha$ represents the volume $V$, the second term of the right-hand side of (12) becomes $\beta P d V$ with the pressure $P=-\langle\partial H / \partial V\rangle_{E, \alpha}^{\mathrm{mc}}$. In general, the relation (12) guarantees the consistency with thermodynamics.

In the following argument, we consider the quasistatic change in $\alpha$. This is realized by choosing $\alpha(t)=\bar{\alpha}(\epsilon t)$, where the functional form of $\bar{\alpha}$ is independent of $\epsilon$, introducing $\tau=\epsilon t$ and taking the quasistatic limit $\epsilon \rightarrow 0$ 
with $\tau_{i}=\epsilon t_{i}$ and $\tau_{f}=\epsilon t_{f}$ fixed. Indeed, $d \alpha / d t=$ $\epsilon d \bar{\alpha} / d \tau=O(\epsilon)$. Now, we take a solution trajectory $\Gamma_{*}(t)$, which is realized in the ideally isolated mechanical system. Then, it determines the time evolution of the energy as $E_{*}(t)=H\left(\Gamma_{*}(t), \alpha(t)\right)$. As the result, the time evolution of the entropy and inverse temperature is also obtained by $S\left(E_{*}(t), \alpha(t)\right)$ and $\beta\left(E_{*}(t), \alpha(t)\right)$, respectively. The adiabatic theorem tells us that $S\left(E_{*}(t), \alpha(t)\right)$ keeps a constant value along almost all solution trajectories in the quasistatic limit [14-19,31]. This means that in the quasistatic limit, almost all solution trajectories with the same initial energy give the same adiabatic curve in the thermodynamic state space $(E, \alpha)$. On the basis of the ideally isolated mechanical system, thus, we have a mechanical description consistent with thermodynamics.

Let us now consider a more realistic situation in which our $N$-particle system enclosed by adiabatic walls is not completely isolated. Then, trajectories of the particles are not solutions to the equation of motion for the Lagrangian (1), because the constituents of the walls may influence the motion of the particles. Even for this case, however, it can be assumed ideally that the $\mathrm{N}$-particle system is thermally isolated (which means adiabatic in thermodynamics) and that the entropy keeps a constant value in quasistatic processes. Motivated by this fact, we try to characterize such phase-space trajectories.

We first identify the condition of phase-space trajectories consistent with quasistatic processes in thermodynamics, which are not necessarily solution trajectories for our Lagrangian (1). We refer to such trajectories as thermodynamically consistent trajectories. Suppose a curve $(\bar{E}(\tau), \bar{\alpha}(\tau)), \quad \tau_{i} \leq \tau \leq \tau_{f}$, in the thermodynamic state space, which corresponds to a quasistatic process in thermodynamics. Here, $\bar{E}(\tau)$ is obtained by $E(t)=$ $\bar{E}(\epsilon t)$, which follows the change of $\bar{\alpha}(\tau)$. Then, for thermodynamically consistent trajectories, the mechanical work $\int d t(d \alpha / d t)(\partial H / \partial \alpha)$ is expected to be equal to the thermodynamic work $\int d t(d \alpha / d t)\langle(\partial H / \partial \alpha)\rangle_{E(t), \alpha(t)}^{\mathrm{mc}}$. We thus define thermodynamically consistent trajectories as those satisfying

$$
\lim _{\epsilon \rightarrow 0} \int_{\tau_{i}^{\prime}}^{\tau_{f^{\prime}}^{\prime}} d \tau \frac{d \bar{\alpha}}{d \tau}\left[\frac{\partial H}{\partial \alpha}-\left\langle\frac{\partial H}{\partial \alpha}\right\rangle_{\bar{E}(\tau), \bar{\alpha}(\tau)}^{\mathrm{mc}}\right]=0
$$

for any time interval $\left[\tau_{i}{ }^{\prime}, \tau_{f}{ }^{\prime}\right]$ such that $\tau_{i} \leq \tau_{i}{ }^{\prime}<\tau_{f}{ }^{\prime} \leq \tau_{f}$. Here, it should be noted that $\partial H / \partial \alpha$ is a rapidly varying function of $\tau$ because it depends on $\Gamma(\tau / \epsilon)$ [32].

Next, we determine the adiabatic condition. Let us fix an adiabatic curve and consider phase-space trajectories that yield the adiabatic curve. From the expression $E(t)=$ $H(\Gamma(t), \alpha(t))$ for any $\Gamma(t)$, we have

$$
\frac{d E}{d t}=\frac{\partial H}{\partial \Gamma} \dot{\Gamma}+\frac{\partial H}{\partial \alpha} \dot{\alpha} .
$$

If the trajectory describes the behavior of a thermally isolated system, the energy changes only through the external control. This property can be represented by

$$
\frac{\partial H}{\partial \Gamma} \dot{\Gamma}=0
$$

This is the condition of the idealized adiabatic wall, which solution trajectories satisfy, of course.

Finally, we check that $S\left(t_{f}\right)=S\left(t_{i}\right)$ holds for thermodynamically consistent trajectories satisfying (16). Here, $S(t) \equiv S(H(\Gamma(t), \alpha(t)), \alpha(t))$ for (11). By using (12) and noting that $d \bar{E} / d \tau=(\partial H / \partial \alpha)(d \bar{\alpha} / d \tau)$ under (16), we express $S\left(t_{f}\right)-S\left(t_{i}\right)=\int_{\tau_{i}}^{\tau_{f}} d \tau(d S(\bar{E}(\tau), \bar{\alpha}(\tau)) / d \tau)$ as

$$
\int_{\tau_{i}}^{\tau_{f}} d \tau \beta \frac{d \bar{\alpha}}{d \tau}\left[\frac{\partial H}{\partial \alpha}-\left\langle\frac{\partial H}{\partial \alpha}\right\rangle_{\bar{E}(\tau), \bar{\alpha}(\tau)}^{\mathrm{mc}}\right] .
$$

Because $\beta(\tau)=\beta(\bar{E}(\tau), \bar{\alpha}(\tau))$ is a slowly varying function of $\tau$, using $\tau_{k}=\left(\tau_{f}-\tau_{i}\right) k / K+\tau_{i}$ with large $K$, (17) may be estimated as

$$
\sum_{k=1}^{K} \beta\left(\tau_{k}\right) \int_{\tau_{k-1}}^{\tau_{k}} d \tau \frac{d \bar{\alpha}}{d \tau}\left[\frac{\partial H}{\partial \alpha}-\left\langle\frac{\partial H}{\partial \alpha}\right\rangle_{\bar{E}(\tau), \bar{\alpha}(\tau)}^{\mathrm{mc}}\right]
$$

with an accuracy of $O(1 / K)$. Then, (18) tends to zero as $\epsilon \rightarrow 0$ due to (14), and (17) is estimated as zero for infinitely large $K$. In the following, this invariance is expressed by the generalized Noether theorem.

Main result.-We now derive the thermodynamic entropy (11) as the Noether invariant $\psi+E \xi$ associated with a transformation $G$. First, we recall that the symmetry exists only if there are $\xi$ and $\psi$ satisfying (6). For the general Lagrangian we study, there are no such $\xi$ and $\psi$ for arbitrary $\hat{q}$ and $\hat{\alpha}$, which is consistent with a fact that the entropy is invariant only in quasistatic adiabatic processes. When we attempt to understand thermodynamic properties, we have to study thermodynamically consistent trajectories. Hence, we can expect that for them there exist $\xi$ and $\psi$ satisfying (6). We shall show this from now. By using the identity

$$
\frac{d E}{d t}=-\mathcal{E} \dot{q}+\frac{\partial E}{\partial \alpha} \dot{\alpha}
$$

we rewrite (6) as

$$
\int_{t_{i}}^{t_{f}} d t \xi\left[\frac{d E}{d t}-\frac{\partial E}{\partial \alpha} \dot{\alpha}\right]=\int_{t_{i}}^{t_{f}} d t \frac{d(\psi+\xi E)}{d t} .
$$

Suppose that $\xi=\Xi(E(q, \dot{q}, \alpha), \alpha)$ and $\psi=\Psi(E(q, \dot{q}, \alpha), \alpha)$ satisfy (20). Then, in the quasistatic limit, (20) becomes 


$$
\int_{\tau_{i}}^{\tau_{f}} d \tau \Xi\left[\frac{d \bar{E}}{d \tau}-\left\langle\frac{\partial H}{\partial \alpha}\right\rangle_{\bar{E}(\tau), \bar{\alpha}(\tau)}^{\mathrm{mc}} \frac{d \bar{\alpha}}{d \tau}\right]=\int_{\tau_{i}}^{\tau_{f}} d \tau \frac{d(\Psi+\Xi \bar{E})}{d \tau}
$$

for thermodynamically consistent trajectories [33]. When there exist $\Xi$ and $\Psi$ satisfying this equation, it should hold for any $\tau_{f}$. This means that the integrand in (21) itself vanishes for each $\tau$, and hence we have

$$
\Xi\left[d \bar{E}-\left\langle\frac{\partial H}{\partial \alpha}\right\rangle_{\bar{E}, \bar{\alpha}}^{\mathrm{mc}} d \bar{\alpha}\right]=d(\Psi+\Xi \bar{E}) .
$$

Let us solve (22). Because the right-hand side is a total derivative of a function of $(E, \alpha)$ [34], the necessary and sufficient condition for the existence of $\Psi(E, \alpha)$ in (22) is given by the integrability condition

$$
\left(\frac{\partial \Xi}{\partial \alpha}\right)_{E}+\frac{\partial}{\partial E}\left(\Xi\left\langle\frac{\partial H}{\partial \alpha}\right\rangle_{E, \alpha}^{\mathrm{mc}}\right)_{\alpha}=0
$$

By using (12), we express the left-hand side as

$$
\frac{\partial}{\partial \alpha}\left[\Xi \beta^{-1}\left(\frac{\partial S}{\partial E}\right)_{\alpha}\right]_{E}-\frac{\partial}{\partial E}\left[\Xi \beta^{-1}\left(\frac{\partial S}{\partial \alpha}\right)_{E}\right]_{\alpha}
$$

Then, we find that the functional determinant $\mid \partial\left(\Xi \beta^{-1}, S\right) /$ $\partial(\alpha, E) \mid$ vanishes. This means that $\Xi=\beta \mathcal{F}(S)$, where $\mathcal{F}$ is an arbitrary function of $S$ [36]. By substituting this into (22), employing (12), and integrating it, we obtain the Noether invariant

$$
\Psi+E \Xi=\int^{S} d S^{\prime} \mathcal{F}\left(S^{\prime}\right)
$$

Note that this is conserved even for thermodynamically consistent adiabatic nonsolution trajectories because the left-hand side of (6) vanishes due to (16).

In particular, we study the Noether invariant $\Psi+E \Xi$ described by an extensive variable for a macroscopic equilibrium system. In this case, the transformation of $\Psi+E \Xi$ for size scaling leads to the result that $\Psi$ is extensive and $\Xi$ is intensive. Because $\beta$ is intensive, $\Xi \beta^{-1}=\mathcal{F}(S)$ becomes a special intensive variable that does not depend explicitly on the extensive work variable $\alpha$ such as the volume $V$.

Let us determine the functional form of $\mathcal{F}(S ; M, N)$, where we explicitly write the dependence on the type of material $M$ and the particle number $N$. The most important property of macroscopic systems is the additivity. As an example, we consider a composite system that consists of two macroscopic subsystems $A$ and $B$ in thermal contact. In the following, we denote physical quantities $Q$ and the type of material $M$ in the subsystem $X$ by $Q_{X}$ and $M_{X}$, respectively, where $X=A$ or $B$.
Now, the time translation $t \rightarrow t+\eta \Xi$ is applied to the composite system. Because the time coordinate is common to the both subsystems, we have $\Xi_{A}=\Xi_{B}$, which is consistent to the intensive nature of $\Xi$. We also have $\beta_{A}=\beta_{B}$ in equilibrium states. These qualities lead to

$$
\mathcal{F}\left(S_{A} ; M_{A}, N_{A}\right)=\mathcal{F}\left(S_{B} ; M_{B}, N_{B}\right) .
$$

From the special property that $\mathcal{F}\left(S_{X} ; M_{X}, N_{X}\right)$ is intensive and independent of $V_{X}$, we can write $\mathcal{F}\left(S_{X} ; M_{X}, N_{X}\right)=$ $\overline{\mathcal{F}}\left(s_{X} ; M_{X}\right)$ with $s_{X} \equiv S_{X} / N_{X}$. Here, if $M_{A}=M_{B}=M$, (26) becomes $\overline{\mathcal{F}}\left(s_{A} ; M\right)=\overline{\mathcal{F}}\left(s_{B} ; M\right)$. Because this holds any $s_{A}$ and $s_{B}$, we conclude that $\overline{\mathcal{F}}(s ; M)=c(M)$, where the constant $c(M)$ depends not on $s$ but on the type of material $M$. Thus, $\mathcal{F}(S ; M, N)=c(M)$ holds generally.

Further, considering a general case $M_{A} \neq M_{B}$ for (26), we have $c\left(M_{A}\right)=c\left(M_{B}\right)$ for any $M_{A}$ and $M_{B}$. That is, $\mathcal{F}=c_{*}$ is a universal constant independent of the type of material. From $c_{*}=\beta^{-1} \Xi$, the universal constant $c_{*}$ has the same dimension as the action, which is known as the Planck constant $\hbar$. Thus, our framework based on classical theory has led to the existence of the Planck constant. Then, we can write $\xi=\hbar \beta$ and $\mathcal{F}=\hbar$, where a dimensionless proportionality constant has been chosen to be unity without loss of generality.

Finally, (25) leads to $\Psi+E \Xi=\hbar S+b \hbar N$, where $b$ is a dimensionless constant. We thus conclude that the thermodynamic entropy $S$ is uniquely characterized as the Noether invariant associated with the transformation $t \rightarrow t+\eta \hbar \beta$ for thermodynamically consistent trajectories [37]. This is the main result of the present Letter.

Concluding remarks.-First of all, we do not have a physical explanation of the symmetry for the real time transformation $t \rightarrow t+\eta \hbar \beta$ yet. It is interesting to find some relation with the fact that the complex time $t+i \hbar \beta$ naturally appears in quantum dynamics with finite temperature. An important point here is that the symmetry is an emergent property in thermodynamic behavior of macroscopic systems, which can build a new bridge between microscopic and macroscopic physics as follows.

One fascinating approach is to generalize this formulation to perfect fluids for interacting particles or relativistic fields, which could provide a more clear view to the symmetry. By restricting the spacetime configurations to those consistent with a local Gibbs distribution at any time, we can find a symmetry leading to the local conservation of the entropy as the Noether charge. It seems reasonable to conjecture that this symmetry is explicitly observed in action functionals for perfect fluids, although the action functionals are not uniquely determined so far [38]. With regard to this point, we also mention a symmetry property announced in Refs. [39,40], which may have some relevance with our theory.

Although our study was motivated by the black hole entropy as the Noether charge [13], it is not clear yet how the present analysis is related to that. Nevertheless, the 
symmetry for $t \rightarrow t+\eta \hbar \beta$ may correspond to that for the Killing parameter translation $v \rightarrow v+\eta \hbar \beta_{H}$, where $\beta_{H}$ is the inverse Hawking temperature [13]. It would be interesting to investigate the connection of our theory with a real-time and microcanonical approach to thermodynamics of gravitational systems [41].

Finally, we have studied the invariant property of the entropy in quasistatic processes. More important is the nondecreasing property of entropy for general timedependent operations. If an initial phase-space point is sampled according to the equilibrium ensemble, this property can be proved [42-44]. It is a challenging problem to combine the symmetry property with the second law of thermodynamics, where the notion of thermodynamically consistent trajectories could be useful.

The authors thank A. Dhar, A. Dechant, M. Hongo, M. Hotta, C. Jarzynski, A. Kundu, R. Loganayagam, C. Maes, and H. Tasaki for helpful discussions. This work was initiated when one of the authors (S. S.) visited Raman Research Institute (RRI). He thanks RRI and International Centre for Theoretical Sciences for the hospitality. The present study was supported by JSPS KAKENHI (No. 25103002 and No. 26610115), by the JSPS Coreto-Core program "Nonequilibrium dynamics of soft-matter and information," and by the RIKEN iTHES Project.

[1] H. B. Callen, Thermodynamics and an Introduction to Thermostatistics (John Wiley and Sons, New York, 1985).

[2] E. H. Lieb and J. Yngvason, Phys. Rep. 310, 1 (1999).

[3] G. Gallavotti, Statistical Mechanics: A Short Treatise (Springer-Verlag, Berlin, Heidelberg, 1999).

[4] T. M. Cover and J. A. Thomas, Elements of Information Theory (John Wiley and Sons, New York, 1991).

[5] M. Li and P. Vitanyi, An Introduction to Kolmogorov Complexity and Its Applications (Springer-Verlag, New York, 1993).

[6] M. A. Nielsen and I. L. Chuang, Quantum Computation and Quantum Information (Cambridge Univ. Press, Cambridge, 2010).

[7] S. W. Hawking, Commun. Math. Phys. 43, 199 (1975).

[8] T. Sagawa and M. Ueda, Phys. Rev. Lett. 102, 250602 (2009).

[9] J. M. R. Parrondo, J. M. Horowitz, and T. Sagawa, Nat. Phys. 11, 131 (2015).

[10] L. Bombelli, R. K. Koul, J. Lee, and R. D. Sorkin, Phys. Rev. D 34, 373 (1986).

[11] M. Srednicki, Phys. Rev. Lett. 71, 666 (1993).

[12] S. Ryu and T. Takayanagi, Phys. Rev. Lett. 96, 181602 (2006).

[13] R. M. Wald, Phys. Rev. D 48, R3427 (1993).

[14] D. V. Anosov, Izv. Akad. Nauk SSSR Ser. Mat. 24, 721 (1960).

[15] T. Kasuga, Proc. Jpn. Acad. 37, 366 (1961); 37, 372 (1961); 37, 377 (1961).

[16] E. Ott, Phys. Rev. Lett. 42, 1628 (1979).
[17] P. Lochak and C. Meunier, Multiphase Averaging for Classical Systems (Springer-Verlag, New York, Berlin, Heidelberg, 1988).

[18] C. Jarzynski, Phys. Rev. Lett. 71, 839 (1993).

[19] M. V. Berry and J. M. Robbins, Proc. R. Soc. A 442, 659 (1993).

[20] See Supplemental Material at http://link.aps.org/ supplemental/10.1103/PhysRevLett.116.140601 for an example of $L(q, \dot{q}, \alpha)$.

[21] A. Trautman, Commun. Math. Phys. 6, 248 (1967).

[22] The original work [23] discussed the case where $\psi=0$, which was generalized to $\psi \neq 0$ by Ref. [24]. Reference [21] investigated the generalization precisely and discussed the property that a solution is mapped to another one by such a transformation. This property was referred to as a dynamical symmetry in Refs. [25] and [26].

[23] E. Noether, Gott. Nachr. 235 (1918); [Transp. Theory Stat. Phys. 1, 186 (1971)].

[24] E. Bessel-Hagen, Math. Ann. 84, 258 (1921).

[25] G. Prince, Bulletin of the Australian Mathematical Society 27, 53 (1983).

[26] W. Sarlet and F. Cantrijn, SIAM Rev. 23, 467 (1981).

[27] The symmetry is mathematically expressed as the invariant property of the contact form in the tangent bundle $[25,26,28]$. This symmetry is called the $d \theta$ symmetry [26] or the Cartan symmetry [25].

[28] M. Crampin, Int. J. Theor. Phys. 16, 741 (1977).

[29] One might notice the absence of the factor $(2 \pi \hbar)^{3 N}$ in (11), but this definition is sufficient when we are not concerned with the additive constant of the entropy.

[30] See Supplemental Material at http://link.aps.org/ supplemental/10.1103/PhysRevLett.116.140601 for the derivation of (ID11).

[31] See Supplemental Material at http://link.aps.org/ supplemental/10.1103/PhysRevLett.116.140601 for the explanation of the adiabatic theorem.

[32] We can check that (14) holds for solution trajectories in the context of the adiabatic theorem. More generally, when the system is considered as a part of a composite system, any quasistatic process can be regarded as the projection of a quasistatic adiabatic process for the composite system to the thermodynamic state space of the system [1]. Then, (14) is satisfied for trajectories of the system. Indeed, we can show this for (nonsolution) trajectories consistent with quasistatic isothermal processes. See Supplemental Material at http://link.aps.org/supplemental/10.1103/PhysRevLett.116 .140601 for more details.

[33] See Supplemental Material at http://link.aps.org/ supplemental/10.1103/PhysRevLett.116.140601 for the derivation of (21).

[34] $\Xi$ in the left-hand side of (22) plays a role of the integrating factor in the Caratheodory theory of thermodynamics [35].

[35] B. Bernstein, J. Math. Phys. 1, 222 (1960).

[36] See Supplemental Material at http://link.aps.org/ supplemental/10.1103/PhysRevLett.116.140601 for the derivation of general $\Xi$ satisfying (23).

[37] See Supplemental Material at http://link.aps.org/ supplemental/10.1103/PhysRevLett.116.140601 for an argument on the special case where the parameter $\alpha$ does not depend on time. 
[38] J. D. Brown, Classical Quantum Gravity 10, 1579 (1993).

[39] F. M. Haehl, R. Loganayagam, and M. Rangamani, J. High Energy Phys. 05 (2015) 060.

[40] J. de Boer, M. P. Heller, and N. Pinzani-Fokeeva, J. High Energy Phys. 08 (2015) 086.
[41] J. D. Brown and J. W. York, Jr., Phys. Rev. D 47, 1420 (1993).

[42] A. Lenard, J. Stat. Phys. 19, 575 (1978).

[43] C. Jarzynski, Phys. Rev. Lett. 78, 2690 (1997).

[44] H. Tasaki, arXiv:cond-mat/0009206. 